\title{
ON GENERATION OF SOLUTIONS OF THE BIHARMONIC EQUATION IN THE PLANE BY CONFORMAL MAPPINGS
}

\section{CHA R LES LOEWNER}

Introduction. The study of harmonic functions in the plane is essentially facilitated by the invariance of the Laplace equation

$$
\nabla^{2} u \equiv \frac{\partial^{2} u}{\partial x^{2}}+\frac{\partial^{2} u}{\partial y^{2}}=0
$$

under the group of conformal mappings. The transformations leaving the biharmonic equation

$$
\nabla^{4} u \equiv \frac{\partial^{4} u}{\partial x^{4}}+2 \frac{\partial^{4} u}{\partial x^{2} \partial y^{2}}+\frac{\partial^{4} u}{\partial y^{4}}=0
$$

invariant are much more restricted; they only form the group of similarity transformations in the $(x, y)$-plane. On the other hand, more general transformations leaving the biharmonic equation invariant may be obtained if $u$ is not treated as a scalar which does not change its value under the transformations, but transformations of the more general type

$$
\begin{aligned}
& x^{\prime}=\phi(x, y) \\
& y^{\prime}=\psi(x, y) \\
& u^{\prime}=\chi(x, y) u
\end{aligned}
$$

are permitted. We assume the functions $\phi, \psi$, and $\chi$ to be four times continuously differentiable, and $\chi \neq 0$. That such nontrivial transformations exist follows immediately from the well-known representation of a biharmonic function $u$ in the form

Received June 23, 1952. This paper is a partly simplified version of work done under sponsorship of the Office of Naval Research. See the Technical Report with the same title, No. 13, N6ori-106, Task V (NR-043-992), with two notes by G. Szegö.

Pacific J. Math. 3 (1953), 417-436 


$$
u=h_{1}(x, y) r^{2}+h_{2}(x, y)
$$

with suitable harmonic functions $h_{1}$ and $h_{2}$, and $r^{2}=x^{2}+y^{2}$. If a transformation by reciprocal radii is applied, which in polar coordinates is given by

$$
r^{\prime}=\frac{1}{r}, \theta^{\prime}=\theta
$$

and $u$ is transformed according to the formula

$$
u^{\prime}=\frac{1}{r^{2}} u,
$$

then $u^{\prime}$ becomes

$$
u^{\prime}=h_{1}^{\prime}\left(x^{\prime}, y^{\prime}\right)+h_{2}^{\prime}\left(x^{\prime}, y^{\prime}\right) r^{\prime 2},
$$

with $h_{1}^{\prime}$ and $h_{2}^{\prime}$ being the harmonic functions of $x^{\prime}, y^{\prime}$ obtained from $h_{1}$ and $h_{2}$ by the transformation (4a). This shows that $u^{\prime}$ is biharmonic in $x^{\prime}$ and $y^{\prime}$.

By combination of the transformation obtained with arbitrary similarities, more general transformation of type (2) may be obtained. In order to write them in a simple way we set

$$
z=x+i y, z^{\prime}=x^{\prime}+i y^{\prime}
$$

One sees easily that the composed transformations can be written in one of the following forms:

$$
\begin{aligned}
& z^{\prime}=\frac{\alpha z+\beta}{\gamma z+\delta}, u^{\prime}=k\left|\frac{d z^{\prime}}{d z}\right| u \\
& z^{\prime}=\frac{\alpha \bar{z}+\beta}{\gamma \bar{z}+\delta}, u^{\prime}=k\left|\frac{d z^{\prime}}{d \bar{z}}\right| u .
\end{aligned}
$$

The constants $\alpha, \beta, \gamma, \delta, k$ are only subjected to the conditions

$$
\left|\begin{array}{c}
\alpha \beta \\
\gamma \delta
\end{array}\right| \neq 0, k \neq 0, k \text { real } .
$$

Each Moebius transformation in the $(x, y)$-plane may, therefore, be extended to a transformation in the $(x, y, u)$-space leaving the biharmonic equation invariant. The extended transformations are analogues of those introduced by 
W. Thomson in his study of the Laplace equation in 3-space.

In the first section of this paper we shall show that formulas $\left(5^{\prime}\right)$ and $\left(5^{\prime \prime}\right)$ represent the only transformations of type (2) leaving the biharmonic equation invariant. They form a group $M$ in the $(x, y, u)$-space depending on seven real parame ters.

The introduction of $M$ has the advantage that if a problem concerning the biharmonic equation is solved for a domain $B$ of the $(x, y)$-plane, it can also be solved for any domain $B^{\prime}$ obtained from $B$ by a Moebius transformation. A further advantage consists in the possibility of introducing domains having $z=\infty$ in the interior or on the boundary. All definitions regarding the behavior of a biharmonic function $u$ at $z=\infty$ are obtained by using one of the transformations (5) transforming $z=\infty$ into a finite point $z^{\prime}=a^{\prime}$, and considering the transformed biharmonic function $u^{\prime}$ at $z^{\prime}=a^{\prime}$. For example, $u$ is called regular at $z=\infty$ if $u^{\prime}$ is regular at $z^{\prime}=a^{\prime}$. Also the concept of a biharmonic Green's function $\Gamma(x, y)$ with the boundary conditions $u=0$ and $\partial u / \partial n=0$ requiring that $u$ and the normal derivative are zero on the boundary (Green's function of the clamped plate) may be extended to the case where the domain considered contains $z=\infty$ in its interior, and $\propto$ should be the pole of $\Gamma$. The singular part of $\Gamma$, belonging to a finite pole $a^{\prime}$, is given by $r^{\prime 2} \log r^{\prime}, r^{\prime}$ denoting the distance of $z^{\prime}=x^{\prime}+i y^{\prime}$ from $a^{\prime}$. By using a transformation (5) transforming $a^{\prime}$ into infinity, one obtains a biharmonic function satisfying the same boundary conditions in the transformed domain whose singular part at $z=\infty$ is $-c \log r$, with a positive constant $c$, and $r$ representing the distance of $z$ from $z=0$ or from any other fixed point of the $z$-plane. In order to make the definition definite we set $c=1$.

This extension of the concept of Green's function will be utilized in $\S 2$, which is concerned with a question of Hadamard [3] regarding the sign of the Green's function. He asked whether it may oscillate in sign. R. J. Duffin [1] indicated that the answer is affirmative by constructing solutions of the biharmonic Poisson equation

$$
\nabla^{4} u=\rho(x, y)
$$

in an infinite straight strip satisfying the boundary conditions

$$
u=0, \frac{\partial u}{\partial n}=0
$$

which oscillate in sign although $\rho$ is positive. In $\S 2$ simple examples of domains 
bounded by analytic Jordan curves are constructed in which Green's function for suitable choice of the pole may oscillate in sign. Other examples were found by G. Szegö [4] and P. R. Garabedian [2].

There are indications that in the exterior of a convex curve with the pole at infinity a change of the sign of the Green's function cannot occur. In the last part of $\$ 2$ we prove only that this conjecture is equivalent to positivity of the harmonic function $\nabla^{2} \Gamma$.

The fact that the biharmonic equation is absolutely invariant only under the group of similarities does not exclude the possibility that for an individual biharmonic function $u$ other conformal mappings exist which transform $u$ into a new biharmonic function. Indeed, we shall show in $\S 1$ that in general there exists a one-parameter family of conformal mappings which are not similarities and which transform $u$ into biharmonic functions. In particular one can construct in this way from one Green's function a one-parameter family of Green's functions of nonsimilar domains. (Only the case of a circle has to be excluded here.) This also will be discussed in $\$ 1$ and applied in $\$ 2$.

1. Transformations of biharmonic functions. We shall prove that the transformations of type (2) leaving the biharmonic equation invariant form the group $M$ described by equations (5). All transformations are assumed to be one-to-one and four times continuously differentiable, and the Jacobian shall never vanish.

We make use of the well-known fact that the biharmonic equation is the Euler-Lagrange equation of the variational problem

$$
\delta \iint\left(\nabla^{2} u\right)^{2} d x d y=0
$$

If the integral of (6) is subjected to a transformation of type (2), an integral in the $\left(x^{\prime}, y^{\prime}\right)$-plane must be obtained whose Euler-Lagrange equation must again be the biharmonic equation

$$
\nabla^{\prime 4} u^{\prime}=0
$$

The new integrand is a quadratic expression in the second derivatives of $u^{\prime}$ with respect to $x^{\prime}$ and $y^{\prime}$, and the second degree terms are evidently given by

$$
\begin{aligned}
& \frac{1}{x^{2}}\left|J\left(\begin{array}{cc}
x & y \\
x^{\prime} y^{\prime}
\end{array}\right)\right|\left\{\frac{\partial^{2} u^{\prime}}{\partial x^{\prime 2}}\left[\left(\frac{\partial x^{\prime}}{\partial x}\right)^{2}+\left(\frac{\partial x^{\prime}}{\partial y}\right)^{2}\right]\right. \\
& \left.+2 \frac{\partial^{2} u^{\prime}}{\partial x^{\prime} \partial y^{\prime}}\left[\frac{\partial x^{\prime}}{\partial x} \frac{\partial y^{\prime}}{\partial x}+\frac{\partial x^{\prime}}{\partial y} \frac{\partial y^{\prime}}{\partial y}\right]+\frac{\partial^{2} u^{\prime}}{\partial y^{\prime 2}}\left[\left(\frac{\partial y^{\prime}}{\partial x^{\prime}}\right)^{2}+\left(\frac{\partial y^{\prime}}{\partial y}\right)^{2}\right]\right\}^{2},
\end{aligned}
$$


$J$ being the Jacobian of the transformation from the $\left(x^{\prime}, y^{\prime}\right)$-plane into the $(x, y)$-plane.

Already from the expression (7) one can derive the fourth order terms of the Euler-Lagrange equation, which by assumption is again the biharmonic equation. This leads by a simple computation to the equations

$$
\begin{array}{r}
\left(\frac{\partial x^{\prime}}{\partial x}\right)^{2}+\left(\frac{\partial x^{\prime}}{\partial y}\right)^{2}=\left(\frac{\partial y^{\prime}}{\partial x}\right)^{2}+\left(\frac{\partial y^{\prime}}{\partial y}\right)^{2}, \\
\frac{\partial x^{\prime}}{\partial x} \frac{\partial y^{\prime}}{\partial x}+\frac{\partial x^{\prime}}{\partial y} \frac{\partial y^{\prime}}{\partial y}=0,
\end{array}
$$

and we see that the mapping must be conformal.

In order to obtain further conditions on the transformation, we specialize $u$ to an arbitrary harmonic function of $x$ and $y$. Since it is then also a harmonic function of $x^{\prime}$ and $y^{\prime}$, we have

$$
\nabla^{\prime 2} u^{\prime}=u \nabla^{\prime 2} x+2\left\{\frac{\partial \chi}{\partial x^{\prime}} \frac{\partial u}{\partial x^{\prime}}+\frac{\partial x}{\partial y^{\prime}} \frac{\partial u}{\partial y^{\prime}}\right\}
$$

and further,

$$
\begin{aligned}
0=\nabla^{\prime 4} u^{\prime} & =u \nabla^{\prime 4} x+4\left\{\frac{\partial \nabla^{\prime 2} \chi}{\partial x^{\prime}} \frac{\partial u}{\partial x^{\prime}}+\frac{\partial \nabla^{\prime 2} \chi}{\partial y^{\prime}} \frac{\partial u}{\partial y^{\prime}}\right\} \\
& +4\left\{\frac{\partial^{2} \chi}{\partial x^{\prime 2}} \frac{\partial^{2} u}{\partial x^{\prime 2}}+2 \frac{\partial^{2} \chi}{\partial x^{\prime} \partial y^{\prime}} \frac{\partial^{2} u}{\partial x^{\prime} \partial y^{\prime}}+\frac{\partial^{2} \chi}{\partial y^{\prime 2}} \frac{\partial^{2} u}{\partial y^{\prime 2}}\right\} .
\end{aligned}
$$

Since $\nabla^{\prime 2} u=0$ represents the only relation between the derivatives of $u^{\prime}$ with respect to $x^{\prime}$ and $y^{\prime}$ up to the second order, we may conclude. from $\left(9^{\prime \prime}\right)$ that

$$
\frac{\partial^{2} \chi}{\partial x^{\cdot 2}}=\frac{\partial^{2} \chi}{\partial y^{\prime 2}}, \frac{\partial^{2} \chi}{\partial x^{\prime} \partial y^{\prime}}=0
$$

The only functions satisfying these conditions are those of the form

$$
\chi=c_{0}\left(x^{\prime 2}+y^{\prime 2}\right)+2 c_{1} x^{\prime}+2 c_{2} y^{\prime}+c_{3}
$$

$$
\left(c_{0}, c_{1}, c_{2}, c_{3} \text { arbitrary constants }\right) .
$$


Application of the same considerations to the inverse transformation leads to a similar formula for the reciprocal of $\chi$ :

$$
\frac{1}{\chi}=c_{0}^{\prime}\left(x^{2}+y^{2}\right)+2 c_{1}^{\prime} x+2 c_{2}^{\prime} y+c_{3}^{\prime},
$$

with suitable constants $c_{0}{ }^{\prime}, c_{1}{ }^{\prime}, c_{2}{ }^{\prime}, c_{3}{ }^{\prime}$. Consider now first the case of a nonconstant $\chi$. According to $\left(11^{\prime}\right)$ and $\left(11^{\prime \prime}\right)$, the level lines of $\chi$ are in both planes systems of concentric circles each of which may degenerate into a system of parallel lines. But a conformal mapping transforming such systems into each other must be, as is well known, a proper or improper lioebius transformation.

In the case of a constant $\chi$ we may proceed as follows: We compose the transformation with one of the transformations (5) having a nonconstant $\chi$, and apply to the composed transformation the previous result saying that now the $(x, y)$-plane is subject to a Moebius transformation. Using the group property of the Moebius transformation, we conclude that also the original transformation of the $(x, y)$-plane is a Moebius transformation.

We now have to investigate how the coefficients $c_{i}$ in $\chi$ depend on the Moebius transformation to which the $(x, y)$-plane is subjected. Since we already know the transformations ( 5 ), it is sufficient to consider only the identity transformation. We know already that $\chi$ must have the form

$$
\chi=c_{0}\left(x^{2}+y^{2}\right)+2 c_{1} x+2 c_{2} y+c_{3},
$$

and multiplication of any biharmonic function $u$ by $\chi$ must again lead to a biharmonic function. Setting

$$
u=x^{2}+y^{2}
$$

gives immediately the result $c_{0}=0$. Setting further

$$
u=x\left(x^{2}+y^{2}\right) \text { or } u=y\left(x^{2}+y^{2}\right)
$$

gives then $c_{1}=c_{2}=0$, and we see that $\chi$ must be a constant. We have thus arrived at:

THEOREM 1. The most general transformations of type (2) which leave the biharmonic equation invariant are represented by formulas $\left(5^{\prime}\right)$ and $\left(5^{\prime \prime}\right)$.

As was already mentioned in the introduction, there exist in general for an 
individual biharmonic function $u(x, y)$ conformal mappings which are not similarities, and which transform $u$ again into a biharmonic function. In order to derive them we make use of the well-known Goursat representation of a biharmonic function,

$$
u=\Re\{\bar{z} p(z)+q(z)\},
$$

where $p(z)$ and $q(z)$ are analytic functions of $z=x+i y$, and the symbol $\Re$ indicates the real part of the quantity in parentheses. The representation is unique modulo a change of $p$ and $q$ into

$$
p_{1}=p+a+i c z, q_{1}=q-\bar{a} z+i d
$$

$(a, c, d$ constants, $c$ and $d$ real $)$.

We write further the Laplacian in the more convenient form

$$
\nabla^{2} u=4 \frac{\partial^{2} u}{\partial z \partial \bar{z}}
$$

Without loss of generality we may restrict our attention to proper conformal mapping. Let

$$
z^{\prime}=f(z)
$$

be such a mapping transforming $u$ into a biharmonic function in the $\left(z^{\prime}=x^{\prime}+i y^{\prime}\right)$ plane. We have

(16') $\frac{\partial^{2} u}{\partial z^{\prime} \partial \bar{z}^{\prime}}=\frac{\partial^{2} u}{\partial z \partial \bar{z}} \frac{d z}{d z^{\prime}} \frac{d \bar{z}}{d \bar{z}^{\prime}}=\Re\left\{\frac{d p}{d z} \frac{d z}{d z^{\prime}} \frac{d \bar{z}}{d \bar{z}^{\prime}}\right\}=\Re\left\{\frac{d p}{d z^{\prime}} \frac{d \bar{z}}{d \bar{z}^{\prime}}\right\}$

and

$$
\frac{\partial^{4} u}{\partial z^{\cdot 2} \partial \bar{z}^{\cdot 2}}=\Re\left\{\frac{d^{2} p}{d z^{\cdot 2}} \frac{d^{2} \bar{z}}{d \bar{z}^{\prime 2}}\right\},
$$

and, therefore,

$$
\Re\left\{\frac{d^{2} p}{d z^{\prime 2}} \frac{d^{2} \bar{z}}{d \bar{z}^{\prime 2}}\right\}=0 \text {. }
$$


Excluding now the trivial case of linear mappings characterized by

$$
\frac{d^{2} z}{d z^{\cdot 2}} \equiv 0
$$

we conclude from (16) that

$$
\frac{d^{2} p}{d z^{\prime 2}}=i c \frac{d^{2} z}{d z^{\prime 2}} \quad(c \text { a real constant })
$$

and hence

$$
p=i c z+\alpha z^{\prime}+\beta \quad(\alpha, \beta \text { constants })
$$

We may exclude also the possibility $\alpha=0$, since then $p=i c z+\beta$ and according to (12) our function $u$ is harmonic. But in this case (15) may be any conformal mapping. If $\alpha \neq 0$ we have

$$
z^{\prime}=a(p-i c z)+b \quad(a, b, c \text { constants, } a \neq 0, c \text { real }) .
$$

We have thus arrived at:

THEOREM 2: A proper conformal mapping transforming a given biharmonic but not harmonic function $u$ with the Goursat representation (12) again into a biharmonic function is either a similarity or one of the transformations (19).

REMARK 1. For the functions $u$ with constant Laplacian $\nabla^{2} u$, the mappings (19) coincide with the similarity mappings, and these are the only biharmonic functions of this type.

REMARK 2. By combination of the transformations (19) with transformations of type (5), more general mappings may be obtained transforming $u$ into a biharmonic function.

2. A question of Hadamard regarding the sign of Green's function of the clamped plate. As was already discussed in the introduction, Hadamard asked whether Green's function of the clamped plate may change its sign. We shall construct here very elementary examples showing that this is the case. In order not to interrupt further considerations, we shall first derive several simple lemmas which will be used in our constructions.

Consider first a finite domain $B$, and let $\Gamma\left(z_{1}, z_{2}\right)$ be its biharmonic 
Green's function now considered as function of a pair of points $z_{1}$ and $z_{2}$ in $B .^{1}$ Because of the well-known symmetry

$$
\Gamma\left(z_{1}, z_{2}\right)=\Gamma\left(z_{2}, z_{1}\right)
$$

it is irrelevant which of the points is considered as pole. We have:

LEMMA 1. For any choice of $m$ points $z_{1}, z_{2}, \ldots, z_{m}$ in $B$, the determinant

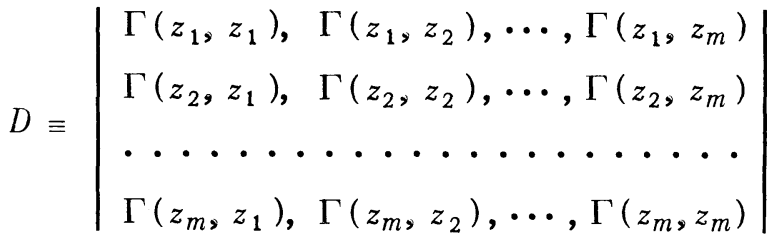

satisfies

$$
D \geq 0
$$

This is an immediate consequence of the well-known fact that $\Gamma\left(z_{1}, z_{2}\right)$ represents a positive definite kernel.

In particular, $\Gamma(a, a) \geq 0$; but the equality sign cannot hold since then the inequality

$$
\left|\begin{array}{cc}
\Gamma(a, a) & \Gamma(a, z) \\
\Gamma(z, a) & \Gamma(z, z)
\end{array}\right|=-\Gamma^{2}(a, z) \geq 0
$$

would lead to $\Gamma(a, z)=0$ for all $z$ in $B$, which is evidently impossible. We have, therefore:

Lemma $2 .^{2}$ For all points $z$ in $B$, we have

$$
\Gamma(z, z)>0 \text {. }
$$

We assume now $B$ to contain $\infty$ in its interior, and state, for its Green's function with the pole at infinity, which we will call $\Gamma(z)$ :

${ }^{1}$ It is, for example, sufficient to assume the boundary of $B$ to be three times continuously differentiable to ensure the existence of $\Gamma$.

${ }^{2}$ Hadamard ascribes the first proof of inequality (21) to M. Boggio. His formula for the variation of $\Gamma(z, z)$ on p. 28 of the already quoted paper also implicitly contains a proof. 
Lemma 3. $\Gamma(z)$ can be represented in the neighborhood of $z=\infty$ in the form

$$
\begin{aligned}
\Gamma(z)=-\log r+a_{0} r^{2}+2 a_{1} x+2 a_{2} y & +\Gamma_{1}(z) \\
& \left(r=|z|, a_{0}, a_{1}, a_{2} \text { constants }\right),
\end{aligned}
$$

with a remainder $\Gamma_{1}$ bounded in the neighborhood of $z=\infty$, and the constant $a_{0}$ is positive.

Proof. If we apply to $\Gamma(z)$ the transformation $\left(5^{\prime}\right)$ corresponding to $z^{\prime}=1 / z$, $\Gamma(z)$ changes into Green's function $\Gamma^{\prime}\left(z^{\prime}\right)$ of the transformed domain $B^{\prime}$ with the pole in $z^{\prime}=0$. But for $\Gamma^{\prime}$ we have

$$
\Gamma^{\prime}\left(z^{\prime}\right)=r^{\prime 2} \log r^{\prime}+a_{0}+2 a_{1} x^{\prime}+2 a_{2} y^{\prime}+\cdots,
$$

the dots indicating quantities of at least second order. The constant $a_{0}$ is positive by Lemma 2. Transforming back, we obtain the contents of Lemma 3.

We introduce now the Goursat representation of $\Gamma$, writing it in the form

$$
\Gamma(z)=\Re\{\bar{z} p(z)\}-h,
$$

where $p(z)$ is analytic and $h$ harmonic. ${ }^{3}$ From Lemma 3 we can easily conclude that the free constants in the choice of $p$ and $h$ can be selected so that the following conditions are satisfied:

(a) The function $p(z)$ has at infinity a simple pole with a positive $p^{\prime}(\infty)$.

(b) The function $h$ differs from $\log r$ by a harmonic function in $B$, regular also at infinity.

By the conditions (a) and (b), the functions $p$ and $h$ are uniquely determined.

We shall now derive properties of $p(z)$ characterizing it independently of $h$. We use the analytic function

$$
w(z)=2 \frac{\partial h}{\partial z} .
$$

\footnotetext{
${ }^{3}$ We assume from now on that $B$ is simply connected, in which case $p(z)$ is always single-valued.
} 
Its expansion at infinity starts with the term $1 / z$. From (23) and the boundary conditions satisfied by $\Gamma$ we conclude that, on the boundary of $B$,

$$
0=\frac{\partial \Gamma}{\partial z}=\frac{1}{2}\left\{\overline{p(z)}+\frac{d p}{d z} \bar{z}\right\}-\frac{\partial h}{\partial z} .
$$

This equation evidently completely expresses the boundary conditions on $\Gamma$ modulo an additive constant. We have proved, therefore:

LEMma 4. The function $p(z)$ is characterized by the two properties:

(a) It has at infinity a simple pole with a positive derivative $p^{\prime}(\infty)$.

(b) The function

$$
\overline{p(z)}+\frac{d p}{d z} \bar{z}
$$

coincides on the boundary of $B$ with a function which is analytic in $B$ and whose expansion at $\infty$ starts with $1 / z$.

We know from Theorem 2 that $p(z)$ maps our domain $B$ onto a domain $B_{1}$ (not necessarily schlicht) with preservation of the Green's function with pole at infinity. In order to bring Lemma 4 into a form in which $B$ and $B_{1}$ play a symmetric role, we introduce the function $z=g(\zeta)$ which maps the exterior of the unit circle $|\zeta|=1$ onto $B$ so that

$$
g(\infty)=\infty, g^{\prime}(\infty)>0 .
$$

In a similar way,

$$
f(\zeta)=p(g(\zeta))
$$

maps the exterior of $|\zeta|=1$ onto the domain $B_{1}$, and we have again

$$
f(\propto)=\infty, f^{\prime}(\infty)>0 .
$$

Lemma 4 can now be expressed by saying that

$$
\overline{f(\zeta)}+\frac{d f}{d \zeta} \frac{d \zeta}{d z} \overline{g(\zeta)}
$$

coincides on $|\zeta|=1$ with the boundary values of a function analytic in $|\zeta|>1$ 
whose Taylor expansion at infinity starts with the term $\left(g^{\prime}(\infty) \zeta\right)^{-1}$. Multiplication with $d z / d \zeta$ leads, therefore, to:

LEMMA 5. The function $f(\zeta)$ is characterized by the following properties:

(a) It is analytic in $|\zeta|>1$ and has at infinity a simple pole with $f^{\prime}(\infty)>0$.

(b) The function

$$
\overline{f(\zeta)} \frac{d g(\zeta)}{d \zeta}+\overline{g(\zeta)} \frac{d f(\zeta)}{d \zeta}
$$

coincides on $|\zeta|=1$ with the boundary values of a function $\omega(\zeta)$ analytic in $|\zeta|>1$ whose expansion at infinity starts with the term $1 / \zeta$.

As soon as $f(\zeta)$ is determined, it is easy to construct the Green's function by using equation (24). It gives, after transformation into the $\zeta$-plane,

$$
2 \frac{\partial h}{\partial \zeta}=\omega(\zeta) \quad(|\zeta| \geq 1)
$$

An integration of $\omega(\zeta)$ determines $h$ modulo an additive constant which is to be adjusted to the boundary condition $\Gamma=0$.

Lemma 5 will now be utilized to find simple examples of domains whose Green's function oscillates in sign. The simplest choice of $g(\zeta)$ one might try would be

$$
g(\zeta)=\zeta+\frac{\beta_{1}}{\zeta} \quad\left(\left|\beta_{1}\right| \leq 1\right)
$$

which maps $|\zeta| \geq 1$ onto the exterior of an ellipse or, in the limiting case $\left|\beta_{1}\right|=1$, onto a slit domain. In this case, one verifies easily that

$$
f(\zeta)=\frac{\zeta-\beta_{1} / \zeta}{2\left(\beta_{1} \bar{\beta}_{1}+1\right)}
$$

and a simple computation gives, for $\Gamma$ written as function of $\zeta$, the expression

$$
\Gamma=\frac{1}{2\left(1+\beta_{1} \bar{\beta}_{1}\right)}\left(\rho^{2}-\frac{\beta_{1} \bar{\beta}_{1}}{\rho^{2}}-1+\beta_{1} \bar{\beta}_{1}\right)-\log \rho,(|\zeta|=\rho, \rho \geq 1) .
$$

But one verifies easily that $\Gamma$ is here always positive. We try, therefore, 
for $g(\zeta)$ an expression ${ }^{4}$

$$
g(\zeta)=\zeta+\frac{\beta_{1}}{\zeta}+\frac{\beta_{2}}{\zeta^{2}}
$$

and shall show that for suitable choice of the constants $\beta_{1}$ and $\beta_{2}$ we obtain a schlicht map of $|\zeta| \geq 1$ on a domain $B$ with a Green's function whose sign oscillates.

First we shall show that the corresponding $f(\zeta)$ is of the form

$$
f(\zeta)=c\left\{\zeta+\alpha_{0}+\frac{\alpha_{1}}{\zeta}+\frac{\alpha_{2}}{\zeta^{2}}\right\}, c>0 .
$$

In order to verify this we introduce the analytic functions

$$
\begin{gathered}
\tilde{g}(\zeta)=\frac{1}{\zeta}+\bar{\beta}_{1} \zeta+\bar{\beta}_{2} \zeta^{2}, \\
\tilde{f}(\zeta)=c\left\{\frac{1}{\zeta}+\bar{\alpha}_{0}+\bar{\alpha}_{1} \zeta+\bar{\alpha}_{2} \zeta^{2}\right\},
\end{gathered}
$$

which coincide on $|\zeta|=1$ with $\overline{g(\zeta)}$ and $\overline{f(\zeta)}$, respectively. According to Lemma 5 , we have to show that the constants $c, \alpha_{0}, \alpha_{1}, \alpha_{2}$ can be chosen in such a way that

$$
\omega(\zeta)=\tilde{f}(\zeta) \frac{d g}{d \zeta}+\tilde{g}(\zeta) \frac{d f}{d \zeta}
$$

has an expansion in $1 / \zeta$ starting with the term $1 / \zeta$. We have

$$
\begin{aligned}
\omega(\zeta) & =c\left\{\frac{1}{\zeta}+\bar{\alpha}_{0}+\bar{\alpha}_{1} \zeta+\bar{\alpha}_{2} \zeta^{2}\right\}\left\{1-\frac{\beta_{1}}{\zeta^{2}}-\frac{2 \beta_{2}}{\zeta^{3}}\right\} \\
& +c\left\{\frac{1}{\zeta}+\bar{\beta}_{1} \zeta+\bar{\beta}_{2} \zeta^{2}\right\}\left\{1-\frac{\alpha_{1}}{\zeta^{2}}-\frac{2 \alpha_{2}}{\zeta^{3}}\right\},
\end{aligned}
$$

or

${ }^{4} \mathrm{~N}$. Mouskhelichvili gave a general procedure telling how to construct Green's func tion of a domain whose mapping function $g(\zeta)$ is rational. We have to compute it in cas (30) in all details. See [4]. N. Mouskhelichvili, Application des intégrales analogues c celles de Cauchy a quelques problème de la physique mathématique, Tiflis, 1922. 


$$
\begin{aligned}
\left(34^{\prime}\right) \omega(\zeta)=c & \left\{\left(\overline{\alpha_{2}}+\overline{\beta_{2}}\right) \zeta^{2}+\left(\bar{\alpha}_{1}+\bar{\beta}_{1}\right) \zeta-\left(\overline{\alpha_{2}} \beta_{1}+\overline{\beta_{2}} \alpha_{1}-\bar{\alpha}_{0}\right)\right. \\
& \left.-\frac{2 \bar{\alpha}_{2} \beta_{2}+2 \bar{\beta}_{2} \alpha_{2}+\bar{\alpha}_{1} \beta_{1}+\bar{\beta}_{1} \alpha_{1}-2}{\zeta}+\cdots\right\} .
\end{aligned}
$$

The conditions on $f(\zeta)$ are, therefore, satisfied if

$$
\alpha_{1}+\beta_{1}=0, \alpha_{2}+\beta_{2}=0, \alpha_{0}=\bar{\alpha}_{1} \beta_{2}+\overline{\beta_{1}} \alpha_{2},
$$

and

$$
c\left(2 \bar{\alpha}_{2} \beta_{2}+2 \bar{\beta}_{2} \alpha_{2}+\bar{\alpha}_{1} \beta_{1}+\bar{\beta}_{1} \alpha_{1}-2\right)=-1,
$$

which gives

$$
\alpha_{1}=-\beta_{1}, \alpha_{2}=-\beta_{2}, \alpha_{0}=-2 \overline{\beta_{1}} \beta_{2},
$$

and

$$
c^{-1}=2\left(1+\beta_{1} \bar{\beta}_{1}+2 \beta_{2} \bar{\beta}_{2}\right) .
$$

Our function $f(\zeta)$ is, therefore, given by

$$
f(\zeta)=\left\{2\left(1+\beta_{1} \bar{\beta}_{1}+2 \beta_{2} \overline{\beta_{2}}\right)\right\}^{-1} \cdot\left\{\zeta-2 \overline{\beta_{1}} \beta_{2}-\frac{\beta_{1}}{\zeta}-\frac{\beta_{2}}{\zeta^{2}}\right\}
$$

In order to obtain $\Gamma$, we have to compute, according to formula (23), the real part of $f(\zeta) \overline{g(\zeta)}$. Using the expression (36), we obtain

$$
\begin{aligned}
c^{-1} f(\zeta) \overline{g(\zeta)} & =\left\{\zeta-2 \bar{\beta}_{1} \beta_{2}-\frac{\beta_{1}}{\zeta}-\frac{\beta_{2}}{\zeta^{2}}\right\}\left\{\bar{\zeta}+\frac{\bar{\beta}_{1}}{\bar{\zeta}}+\frac{\overline{\beta_{2}}}{\overline{\zeta^{2}}}\right\} \\
& =\zeta \bar{\zeta}-2 \bar{\beta}_{1} \beta_{2} \bar{\zeta}+\bar{\beta}_{1} \frac{\zeta}{\bar{\zeta}}-\beta_{1} \frac{\bar{\zeta}}{\zeta}+\bar{\beta}_{2} \frac{\zeta}{\bar{\zeta}^{2}}-2 \frac{\bar{\beta}_{1}^{2} \beta_{2}}{\bar{\zeta}}-\beta_{2} \frac{\bar{\zeta}}{\zeta^{2}} \\
& -\frac{2 \bar{\beta}_{1} \bar{\beta}_{2} \beta_{2}}{\bar{\zeta}^{2}}-\frac{\beta_{1} \bar{\beta}_{1}}{\zeta \bar{\zeta}}+\frac{\beta_{1} \bar{\beta}_{2}}{\zeta \bar{\zeta}^{2}}-\frac{\bar{\beta}_{1} \beta_{2}}{\bar{\zeta} \zeta^{2}}-\frac{\beta_{2} \bar{\beta}_{2}}{\zeta^{2} \bar{\zeta}^{2}},
\end{aligned}
$$

and 


$$
\begin{aligned}
c^{-1} \Re\{f(\zeta) \overline{g(\zeta)}\}= & \rho^{2}-\frac{\beta_{1} \bar{\beta}_{1}}{\rho^{2}}-\frac{\beta_{2} \bar{\beta}_{2}}{\rho^{4}} \\
& -2 \Re\left\{\beta_{1} \bar{\beta}_{2} \zeta+\frac{\beta_{1}^{2} \bar{\beta}_{2}}{\zeta}+\frac{\beta_{1} \beta_{2} \bar{\beta}_{2}}{\zeta^{2}}+\frac{\bar{\beta}_{1} \beta_{2}}{\bar{\zeta} \zeta^{2}}\right\} .
\end{aligned}
$$

We substitute further into the formula $(34)$ for $\omega(\zeta)$ the expressions (35) for $\alpha_{0}, \alpha_{1}, \alpha_{2}$, and obtain

$$
\begin{aligned}
\omega(\zeta) & =c\left\{\frac{1}{\zeta}-2 \beta_{1} \bar{\beta}_{2}-\bar{\beta}_{1} \zeta-\bar{\beta}_{2} \zeta^{2}\right\}\left\{1-\frac{\beta_{1}}{\zeta^{2}}-\frac{2 \beta_{2}}{\zeta^{3}}\right\} \\
& +c\left\{\frac{1}{\zeta}+\bar{\beta}_{1} \zeta+\bar{\beta}_{2} \zeta^{2}\right\}\left\{1+\frac{\beta_{1}}{\zeta^{2}}+\frac{2 \beta_{2}}{\zeta^{3}}\right\},
\end{aligned}
$$

or, after a simple computation,

$$
\omega(\zeta)=\frac{1}{\zeta}+c\left\{\frac{2\left(\beta_{1}^{2} \bar{\beta}_{2}+2 \bar{\beta}_{1} \beta_{2}\right)}{\zeta^{2}}+\frac{4 \beta_{1} \beta_{2} \bar{\beta}_{2}}{\zeta^{3}}\right\}
$$

In order to obtain $h$ we may, according to $(27)$, integrate $\omega(\zeta)$ :

(39) $\int \omega(\zeta) d \zeta=\log \zeta-2 c\left\{\frac{\beta_{1}^{2} \bar{\beta}_{2}+2 \bar{\beta}_{1} \beta_{2}}{\zeta}+\frac{\beta_{1} \beta_{2} \bar{\beta}_{2}}{\zeta^{2}}\right\}+C(C$ a constant $)$

Since the real part of (39) must coincide on $|\zeta|=1$ with $\Re\{f(\zeta) \overline{g(\zeta)}\}$, we obtain, by comparison of (37) and (39),

$$
c\left\{1-\beta_{1} \bar{\beta}_{1}-\beta_{2} \bar{\beta}_{2}\right\}=\Re(C),
$$

or

$$
\Re(C)=\frac{1-\beta_{1} \bar{\beta}_{1}-\beta_{2} \bar{\beta}_{2}}{2\left(1+\beta_{1} \bar{\beta}_{1}+2 \beta_{2} \bar{\beta}_{2}\right)}
$$

From the foregoing formulas we finally obtain, by substitution into (23), the following expression for $\Gamma(z)$ in terms of $\zeta$ : 


$$
\begin{aligned}
c^{-1} \Gamma= & \rho^{2}-\frac{\beta_{1} \bar{\beta}_{1}}{\rho^{2}}-\frac{\beta_{2} \bar{\beta}_{2}}{\rho^{4}}-2 \Re\left\{\beta_{1} \bar{\beta}_{2} \zeta-\frac{2 \bar{\beta}_{1} \beta_{2}}{\zeta}+\frac{\bar{\beta}_{1} \beta_{2}}{\rho^{2} \zeta}\right\} \\
& -\frac{1}{c} \log \rho-\left(1-\beta_{1} \bar{\beta}_{1}-\beta_{2} \bar{\beta}_{2}\right),
\end{aligned}
$$

with

$$
c^{-1}=2\left(1+\beta_{1} \bar{\beta}_{1}+2 \dot{\beta}_{2} \bar{\beta}_{2}\right) .
$$

We can now show that the constants $\beta_{1}$ and $\beta_{2}$ can be chosen in such a way that $g(\zeta)$ represents a schlicht mapping of $|\zeta| \geq 1$, and still $\Gamma(\zeta)$ oscillates in sign. Evidently we obtain an oscillating $\Gamma$ if the normal derivative

$$
\left(\frac{\partial^{2} \Gamma}{\partial \rho^{2}}\right)_{\rho=1}
$$

on the unit circle $|\zeta|=1$ becomes negative in some of its points. But we have, from (41),

$$
\begin{aligned}
& c^{-1}\left(\frac{\partial^{2} \Gamma}{\partial \rho^{2}}\right)_{\rho=1}=2-6 \beta_{1} \bar{\beta}_{1}-20 \beta_{2} \bar{\beta}_{2} \\
& -2 \Re\left\{-\frac{4 \bar{\beta}_{1} \beta_{2}}{\zeta}+\frac{6 \bar{\beta}_{1} \beta_{2}}{\zeta}+\frac{2 \bar{\beta}_{1} \beta_{2}}{\zeta}+\frac{4 \bar{\beta}_{1} \beta_{2}}{\zeta}\right\}+2\left(1+\beta_{1} \bar{\beta}_{1}+2 \beta_{2} \bar{\beta}_{2}\right),
\end{aligned}
$$

or

$$
c^{-1}\left(\frac{\partial^{2} \Gamma}{\partial \rho^{2}}\right)_{\rho=1}=4-4 \beta_{1} \bar{\beta}_{1}-16 \beta_{2} \bar{\beta}_{2}-16 \Re \frac{\beta_{1} \bar{\beta}_{2}}{\zeta} \quad(|\zeta|=1) .
$$

This expression becomes negative on the unit circle if

$$
16\left|\beta_{1} \beta_{2}\right|>4-4 \beta_{1} \bar{\beta}_{1}-16 \beta_{2} \bar{\beta}_{2},
$$

or

$$
\left|\beta_{1}\right|+2\left|\beta_{2}\right|>1
$$


The function $g(\zeta)$ represents a schlicht mapping of the exterior of the unit circle if the difference quotient

$$
\frac{g\left(\zeta_{1}\right)-g\left(\zeta_{2}\right)}{\zeta_{1}-\zeta_{2}}
$$

has positive real part there. This is the case if

$$
\left|\beta_{1}\right|+2\left|\beta_{2}\right| \leq 1
$$

But even the exterior of slightly smaller circle is mapped schlicht under this condition if only the cases $\left|\beta_{1}\right|+2\left|\beta_{2}\right|=1$, and $\beta_{1}^{3}$ and $\beta_{2}^{2}$ of equal argument, are excluded. If, therefore, $\left|\beta_{1}\right|+2\left|\beta_{2}\right|=1$, and $\beta_{1}^{3}$ and $\beta_{2}^{2}$ have different arguments, all sufficiently close values $\beta_{1}, \beta_{2}$ give schlicht mappings of $|\zeta| \geq 1$, and can be chosen so that (44) is satisfied. We thus obtain examples of domains where $\Gamma$ oscillates in sign.

We conjecture that, for the exterior of a convex curve and pole at infinity, $\Gamma$ is positive. We shall support this conjecture by proving:

THEOREM 3. For the exterior of a convex curve and pole at infinity the positivity of $\Gamma$ is equivalent to the positivity of $\nabla^{2} \Gamma$.

This is important because $\nabla^{2} \Gamma$ is a harmonic function.

First we shall prove that the positivity of $\Gamma$ implies the positivity of $\nabla^{2} \Gamma$. Assume first that the boundary curve is analytic. Then $\Gamma$ can be analytically continued beyond the boundary curve, and we can speak of derivatives of higher order on the curve itself. From the positivity of $\Gamma$ and the boundary condition it follows immediately that the normal derivative of second order on the boundary satisfies

$$
\frac{\partial^{2} \Gamma}{\partial n^{2}} \geq 0
$$

But the second derivative in the tangential direction is zero again on account of the boundary conditions. We have, therefore, $\nabla^{2} \Gamma \geq 0$ on the boundary. Since $\nabla^{2} \Gamma$ is harmonic and, on account of Lemma 3,

$$
\left[\nabla^{2} \Gamma\right]_{z=\infty}>0
$$

the Laplacian $\nabla^{2} \Gamma$ is positive in the whole domain. 
The condition of analyticity of the boundary curve now can easily be dropped by a limiting process.

We shall now prove the converse: $\nabla^{2} \Gamma>0$ implies $\Gamma>0$. For the proof we need some preliminary considerations regarding the following question: Which differential operators of second order,

$$
\begin{aligned}
v(x, y) & =a_{11}(x, y) \frac{\partial^{2} u}{\partial x^{2}}+2 a_{12}(x, y) \frac{\partial^{2} u}{\partial x \partial y}+a_{22}(x, y) \frac{\partial^{2} u}{\partial y^{2}} \\
& +2 a_{1}(x, y) \frac{\partial u}{\partial x}+2 a_{2}(x, y) \frac{\partial u}{\partial y}+a_{3}(x, y) u,
\end{aligned}
$$

transform an arbitrary biharmonic function $u(x, y)$ into a harmonic function $v(x, y)$ ? The answer is given in:

LEMma 6. The most general operator of the required type is of the form

(46) $v(x, y)=m(x, y) \nabla^{2} u-2\left\{\frac{\partial m(x, y)}{\partial x} \frac{\partial u}{\partial x}+\frac{\partial m(x, y)}{\partial y} \frac{\partial u}{\partial y}\right\}+\nabla^{2} m \cdot u$,

where the function $m(x, y)$ is of the form

$m(x, y)=c_{0}\left(x^{2}+y^{2}\right)+2 c_{1} x+2 c_{2} y+c_{3} \quad\left(c_{0}, c_{1}, c_{2}, c_{3}\right.$ constants $)$.

Proof. We form

(48 $) \quad \nabla^{2} v=a_{11} \frac{\partial^{2} \nabla^{2} u}{\partial x^{2}}+2 a_{12} \frac{\partial^{2} \nabla^{2} u}{\partial x \partial y}+a_{22} \frac{\partial^{2} \nabla^{2} u}{\partial y^{2}}+$ terms of lower order.

Since the only relation between the derivatives of $u$ up to the fourth order is given by $\nabla^{4} u=0$, we see already from $\left(48^{\circ}\right)$ that

$$
a_{12}=0, a_{11}=a_{22} \text {. }
$$

Calling $a_{11}=a_{22}=m$, we can write $(45)$ in the form

$$
v=m \nabla^{2} u+2 a_{1} \frac{\partial u}{\partial x}+2 a_{2} \frac{\partial u}{\partial y}+a_{3} u
$$

From $\left(45^{\circ}\right)$ we obtain 
(48") $\quad \nabla^{2} v=2\left\{\frac{\partial m}{\partial x} \frac{\partial \nabla^{2} u}{\partial x}+\frac{\partial m}{\partial y} \frac{\partial \nabla^{2} u}{\partial y}\right\}+2\left\{a_{1} \frac{\partial \nabla^{2} u}{\partial x}+a_{2} \frac{\partial \nabla^{2} u}{\partial y}\right\}$

+ terms of lower order.

From $\left(48^{\prime \prime}\right)$ we conclude that

$$
a_{1}=-\frac{\partial m}{\partial x}, \quad a_{2}=-\frac{\partial m}{\partial y}
$$

and $\left(45^{\circ}\right)$ can be rewritten as

$$
v=m \nabla^{2} u-2\left\{\frac{\partial m}{\partial x} \frac{\partial u}{\partial x}+\frac{\partial m}{\partial y} \frac{\partial u}{\partial y}\right\}+a_{3} u
$$

From this equation we derive

$$
\begin{array}{r}
\left(48^{\prime \prime \prime)} \quad \nabla^{2} v=\nabla^{2} m \nabla^{2} u-4\left\{\frac{\partial^{2} m}{\partial x^{2}} \frac{\partial^{2} u}{\partial x^{2}}+2 \frac{\partial^{2} m}{\partial x \partial y} \frac{\partial^{2} u}{\partial x \partial y}+\frac{\partial^{2} m}{\partial y^{2}} \frac{\partial^{2} u}{\partial y^{2}}\right\}\right. \\
+a_{3} \nabla^{2} u+\text { term of lower order, }
\end{array}
$$

which gives

$$
\frac{\partial^{2} m}{\partial x^{2}}=\frac{\partial^{2} m}{\partial y^{2}}, \frac{\partial^{2} m}{\partial x \partial y}=0, a_{3}=\nabla^{2} m
$$

and we obtain (45) in the form (46) with $m$ given by an expression (47). We verify easily that for any such choice of $m$ the Laplacian satisfies

$$
\nabla^{2} v=0
$$

Let $B$ now be the exterior of a closed convex curve, and $\Gamma$ its Green's function with the singular point at infinity, and assume that $\nabla^{2} \Gamma>0$ in $B$. Take a straight line which does not penetrate into the interior of the boundary curve. By change of the coordinate system we can make this line the $y$-axis, so that $B$ lies to its left. We now apply Theorem 5 with the special choice $m=x$, and obtain that

$$
v=x \nabla^{2} \Gamma-2 \frac{\partial \Gamma}{\partial x}
$$


is harmonic in $B$. On the boundary of $B$,

$$
\frac{\partial \Gamma}{\partial x}=0, \nabla^{2} \Gamma \geq 0
$$

the latter inequality being a consequence of the assumption that $\nabla^{2} \Gamma>0$ in $B$. Since our domain lies completely in the half-plane $x \leq 0$, we conclude from (51) that $v$ has nonpositive boundary values. From the behavior of $\Gamma$ at $z=\infty$ expressed by Lemma 2, we see that $v$ is regular at infinity. From the extremum properties of harmonic functions we now conclude that $v \leq 0$ in the whole $B$. In particular, on the $y$-axis where $x=0$ we obtain the result

$$
\frac{\partial \Gamma}{\partial x} \geq 0
$$

Let $l$ now be a half line originating in a point $C$ of the boundary curve of $B$ orthogonal to the tangent line at $C$. Laying the $y$-axis perpendicular to $l$ through any of its points, we see that $(52)$ holds in all points of $l$; and, since $\Gamma=0$ at $C$, we arrive at the inequality $\Gamma \geq 0$ along the whole $l$. But the equality cannot hold, for otherwise $\Gamma$ would be zero along a whole segment of $l$ and, since it is analytic, along the whole $l$. This contradicts Lemma 2, which implies that $\Gamma \longrightarrow \infty$ as $z \longrightarrow \infty$. The whole domain $B$ can be covered with half lines having the properties of $l$. The inequality $\Gamma>0$ holds, therefore, in the whole $B$.

\section{REFERENCES}

1. R. J. Duffin, On a question of Hadamard concerning superbiharmonic functions, J. Math. Physics, 27 (1949), 253-258.

2. P. R. Garabedian, A partial differential equation arising in conformal mapping, O.N.R. Technical Report No. 8, N6ori-106, Task V (NR-043-992).

3. J. Hadamard, Mémoire sur le problème d'analyse rélatif à l'équilibre des plaques élastiques encastrées, Mémoire présentée par divers savants à l'Académie des Sciences, ser. 2, 33 (1908), 128 pages.

4. N. Mouskhelichvili, Application des intégrales analogues a celles de Cauchy a quelques problème de la physique mathématique, Tiflis, 1922.

5. G. Szegö, See the paper following the present paper.

\section{STANFORD UNIVERSITY}

